\title{
The resurgent influence of big formula
}

A word was changed during editing of this article (BMJ 2018;362:k3577, doi:10.1136/bmj.k3577) that led to an incorrect statement. It should read "The increasing use of infant formula in low and middle income countries has coincided with a slowing of the fall in infant and maternal mortality." 